\title{
The Laplacian Energy of Conjugacy Class Graph of Some Finite Groups
}

\author{
${ }^{1}$ Rabiha Mahmoud*, ${ }^{2}$ Amira Fadina Ahmad Fadzil, ${ }^{3}$ Nor Haniza Sarmin \\ and ${ }^{4}$ Ahmad Erfanian \\ ${ }^{1,2,3}$ Department of Mathematical Sciences, Universiti Teknologi Malaysia \\ 81310 UTM Johor Bahru, Malaysia \\ ${ }^{4}$ Department of Pure Mathematics, Faculty of Mathematical Sciences, \\ and Center of Excellence in Analysis on Algebraic Structures, \\ Ferdowsi University of Mashhad, Mashhad, Iran \\ *Corresponding author: rabihabirkia@gmail.com
}

Article history

Received: 11 January 2018

Received in revised form: 16 February 2019

Accepted: 4 March 2019

Published on line: 1 April 2019

\begin{abstract}
Let $G$ be a dihedral group and $\Gamma_{G}^{c l}$ its conjugacy class graph. The Laplacian energy of the graph, $L E\left(\Gamma_{G}^{c l}\right)$ is defined as the sum of the absolute values of the difference between the Laplacian eigenvalues and the ratio of twice the edges number divided by the vertices number. In this research, the Laplacian matrices of the conjugacy class graph of some dihedral groups, generalized quaternion groups, quasidihedral groups and their eigenvalues are first computed. Then, the Laplacian energy of the graphs are determined.
\end{abstract}

Keywords dihedral groups; generalized quaternion groups; quasidihedral groups; conjugacy class graph; Laplacian energy; Laplacian matrix; eigenvalues.

Mathematics Subject Classification 05C50, 20C30, $20 \mathrm{~B} 05$.

\section{Introduction}

The definition of the energy of a graph was introduced by Gutman in 1978 [1]. The energy of a graph is defined as the sum of the absolute values of its eigenvalues. It is applied in chemistry to approximate the total electron energy of molecules [2]. In 2006, Gutman and Zhou [3] defined the Laplacian energy of a graph as the sum of the absolute deviations (i.e. distance from the mean) of the eigenvalues of its Laplacian matrix. Similar variants of graph energy were devolved for the signless Laplacian [4], the distance matrix [5] and the incidence matrix [6]. In 2010, Cavers, Fallat, and Kirkard [7] first studied the normalized Laplacian energy of a graph. Laplacian eigenvalues of La graph are the eigenvalues of its Laplacian matrix.

The major contribution of this study is to provide new theoretical results on the Laplacian energy of conjugacy class graph of some finite groups and the formulas for the Laplacian energy of conjugacy class graph has been successfully generalized. In other fields, graphs representing conjugated molecules, the Laplacian energy of the graph $L E(\Gamma)$ is closely related to their total 
$\pi$-electron energy, as calculated within Huckel molecular orbital approximation. In most cases the Laplacian energy can be used to calculate the energy of molecular structures in a much simpler way. The results in this paper revealed more properties and classifications of the groups in terms of conjugacy classes of the elements of the groups.

A graph $\Gamma$ is a finite nonempty set of objects called vertices together with a set of unordered pairs of distinct vertices of $\Gamma$ called the edges [8]. The vertex-set of $\Gamma$ is denoted by $V(\Gamma)$, while the edge-set is denoted by $E(\Gamma)$.

Let $\Gamma$ be a graph with set of vertices $V(\Gamma)=\{1, \ldots, n\}$ and the set of edges $E(\Gamma)=$ $\left\{e_{1}, \ldots, e_{n}\right\}$. The adjacency matrix of $\Gamma$ denoted by $A(\Gamma)$ is an $n \times n$ matrix defined as follows: the rows and the columns of $A(\Gamma)$ are indexed by $V(\Gamma)$. If $i \neq j$, then the $(i, j)-\operatorname{entry}$ of $A(\Gamma)$ is 0 and 1 for nonadjacent vertices $i$ and $j$ respectively. The $(i, i)$-entry of $A(\Gamma)$ is 0 for $i=1, \ldots, n$ [1]. The degree of vertex $i$ is denoted by $d_{\Gamma}(i)$. Let $D(\Gamma)=\operatorname{diag}\left(d_{\Gamma}(1), d_{\Gamma}(2), \ldots, d_{\Gamma}(n)\right)$ be the diagonal matrix of vertex degrees. The Laplacian matrix $L(\Gamma)=D(\Gamma)-A(\Gamma)[9]$.

Suppose $G$ is a finite group. Two elements $a$ and $b$ of $G$ are called conjugate if there exists an element $g \in G$ where $g a g^{-1}=b$. The conjugacy class is an equivalence relation and therefore partition $G$ into some equivalence classes. This means that every element of the group $G$ belongs to precisely one conjugacy class. The equivalence class that contains the element $a \in G$ is $c l(a)=\left\{g a g^{-1}: g \in G\right\}$ and is called the conjugacy class of $a$. The classes $c l(a)$ and $c l(b)$ are equal if and only if $a$ and $b$ are conjugate. The class number of $G$ is the number of distinct (non-equivalent) conjugacy classes and we denote it by $K(G)$.

\section{Preliminaries}

In this section some concepts on the conjugacy class graph that will be used in the following section are presented.

Definition 1 [3] Laplacian energy of a graph is the sum of the absolute deviations (i.e. distance from the mean) of the eigenvalues of its Laplacian matrix.

Definition 2 [10] Let $G$ be a finite group and let $Z(G)$ be the center of $G$. The vertices of the conjugacy class graph of $G$ are non-central conjugacy classes of $G$ i.e. $|V(G)|=K(G)-|Z(G)|$, where $K(G)$ is the class number of $G$. Two vertices are adjacent if their cardinalities are not coprime (i.e. have common factor).

Theorem 1 [11] The conjugacy classes of a dihedral group $D_{2 n}=\langle a, b| a^{n}=b^{2}=1$, $\left.b a b=a^{-1}\right\rangle$, where $n \geq 3$ are as follows, depending on the parity of $n$.

1. For odd $n$ :

$\{1\},\left\{a, a^{-1}\right\}, \ldots,\left\{a^{\frac{n-1}{2}}, a^{-\left(\frac{n-1}{2}\right)}\right\},\left\{a^{i} b, 0 \leq i \leq n-1\right\}$.

2. For even $n$ :

$\{1\},\left\{a, a^{-1}\right\},\left\{a^{2}, a^{-2}\right\}, \ldots,\left\{a^{\frac{n-2}{2}}, a^{-\left(\frac{n-2}{2}\right)}\right\},\left\{a^{\frac{n}{2}}\right\},\left\{a^{2 i} b, 0 \leq i \leq \frac{n-2}{2}\right\}$ and $\left\{a^{2 i+1} b, 0 \leq\right.$ $\left.i \leq \frac{n-2}{2}\right\}$. 
Proposition $1[12]$ Let $D_{2 n}=\left\langle a, b \mid a^{n}=b^{2}=1, b a b=a^{-1}\right\rangle$ be a dihedral group of order $2 n$ where $n \geq 3, n \in \mathbb{Z}^{+}$. Then the conjugacy class graphs of $D_{2 n}$, are as follows:

$$
\Gamma_{D_{2 n}}^{c l}=\left\{\begin{array}{l}
K_{\frac{n-1}{2}} \cup c l(b), \quad \text { if } n \text { odd, } \\
K_{\frac{n+2}{2}}, \text { if } n \text { and } \frac{n}{2} \text { even, } \\
K_{n-1} \cup K_{2}, \quad \text { if } n \text { even and } \frac{n}{2} \text { odd }
\end{array}\right.
$$

Proposition 2 [12] Let $Q_{4 n}$ be a generalized quaternion group of order $4 n$, where $n \geq 2, n \in$ $\mathbb{N}$. Then the conjugacy class graphs of $Q_{4 n}$ are as follows:

$$
\Gamma_{Q_{4 n}}^{c l}=\left\{\begin{array}{l}
K_{n+1}, \text { if } n \text { even, } \\
K_{n-1} \cup K_{2}, \text { if } n \text { odd }
\end{array}\right.
$$

Proposition 3 [12] Let $Q D_{2^{n}}$ be a quasidihedral group of order $2^{n}$, where $n \geq 4, n \in \mathbb{Z}^{+}$. Then the conjugacy class graphs of $Q D_{2^{n}}$ is given as

$$
\Gamma_{Q D^{n}}^{c l}=K_{2^{n-2}+1} .
$$

Proposition 4 [13] The multiplicity of 0 as eigenvalue of $L(\Gamma)$ is equal to the number of connected components of the graph.

Proposition 5 [14] The Laplacian matrix of the complete graph, $K_{n}$ has eigenvalues 0 with multiplicity 1 and $n$ with multiplicity $n-1$.

\section{Main Results}

In this section, we present our main results, namely the Laplacian energy of the conjugacy class graph of some dihedral groups of order $2 n, n \geq 3, n \in \mathbb{Z}^{+}$for $n$ an even integer, generalized quaternion groups and quasidihedral groups. First, we found the Laplacian energy of $\Gamma_{D_{2 n}}^{c l}$ according to the cases in Proposition 2.1.

Theorem 2 Let $D_{2 n}=\left\langle a, b \mid a^{n}=b^{2}=1, b a b^{-1}=a^{-1}\right\rangle$ be a dihedral group of order $2 n$, where both $n$ and $\frac{n}{2}$ are even integers and let $\Gamma_{D_{2 n}}^{c l}$ be its conjugacy class graph. Then, the Laplacian energy of $\Gamma_{D_{2 n}}^{c l}$ is $L E\left(\Gamma_{D_{2 n}}^{c l}\right)=n$.

Proof Consider that $D_{2 n}$ is a dihedral group of order $2 n$, where $n$ and $\frac{n}{2}$ are even integers and $\Gamma_{D_{2 n}}^{c l}$ is its conjugacy class graph. From Proposition 2.1, where $n$ and $\frac{n}{2}$ are even, $\Gamma_{D_{2 n}}^{c l}=K_{\frac{n+2}{2}}$. Hence

$$
\left|V\left(\Gamma_{D_{2 n}}^{c l}\right)\right|=\frac{n+2}{2},\left|E\left(\Gamma_{D_{2 n}}^{c l}\right)\right|=\frac{\frac{n+2}{2}\left(\frac{n+2}{2}-1\right)}{2}=\frac{n(n+2)}{8}
$$

and the Laplacian eigenvalues of $\Gamma_{D_{2 n}}^{c l}$ are $\mu=0$ and $\mu=\frac{n+2}{2}$ with multiplicity $\frac{n}{2}$. By the definition of the Laplacian energy, we have

$$
L E\left(\Gamma_{G}\right)=\sum_{i=1}^{k}\left|\mu_{i}-\frac{2 m}{n}\right|
$$


where $\mu_{i}$ are Laplacian eigenvalues of $\Gamma_{G}, m$ is the number of edges and $n$ is the number of vertices. Thus, replacing $\mu$ by 0 and $\frac{n+2}{2}, m$ by $\frac{n(n+2)}{8}$ and $n$ by $\frac{n+2}{2}$, the Laplacian energy of $\Gamma_{D_{2 n}}^{c l}$ is

$$
L E\left(\Gamma_{D_{2 n}}^{c l}\right)=\left|0-\frac{2 \frac{n(n+2)}{8}}{\frac{n+2}{2}}\right|+\frac{n}{2}\left|\left(\frac{n+2}{2}\right)-\left(\frac{2 \frac{n(n+2)}{8}}{\frac{n+2}{2}}\right)\right|=\left|-\frac{n}{2}\right|+\frac{n}{2}\left|\frac{n+2}{2}-\frac{n}{2}\right|=n .
$$

Theorem 3 Let $D_{2 n}=\left\langle a, b \mid a^{n}=b^{2}=1, b a b^{-1}=a^{-1}\right\rangle$ be a dihedral group of order $2 n$, where $n \geq 3, n \in \mathbb{Z}^{+}, n$ is an even integer and $\frac{n}{2}$ is an odd integer and let $\Gamma_{D_{2 n}}^{c l}$ be its conjugacy class graph. Then, the Laplacian energy of $\Gamma_{D_{2 n}}^{c l}$ is $L E\left(\Gamma_{D_{2 n}}^{c l}\right)=n-2$.

Proof Consider that $D_{2 n}$ is a dihedral group of order $2 n$, where $n$ is even, $\frac{n}{2}$ is odd and $\Gamma_{D_{2 n}}^{c l}$ is its conjugacy class graph. From Proposition 2.1, for $n$ even and $\frac{n}{2}$ odd,

$$
\Gamma_{D_{2 n}}^{c l}=K_{\frac{n-2}{2}} \cup K_{2}
$$

Hence,

$$
\left|V\left(K_{\frac{n-2}{2}}\right)\right|=\frac{n-2}{2},\left|E\left(K_{\frac{n-2}{2}}\right)\right|=\frac{(n-2)(n-4)}{8}
$$

and the Laplacian eigenvalues of $K_{\frac{n-2}{2}}$ are $\mu=0$ and $\mu=\frac{n-2}{2}$ with multiplicity $\frac{n-4}{2}$. By the definition of the Laplacian energy, we have

$$
L E\left(\Gamma_{G}\right)=\sum_{i=1}^{k}\left|\mu_{i}-\frac{2 m}{n}\right|
$$

where $\mu_{i}$ are Laplacian eigenvalues of $\Gamma_{G}, m$ is the number of edges and $n$ is the number of vertices. Thus, replacing $\mu$ by $0, \frac{n-2}{2}$ with multiplicity $\frac{n-4}{2}, m$ with $\frac{(n-2)(n-4)}{8}$ and $n$ with $\frac{n-2}{2}$, the Laplacian energy of $K_{\frac{n-2}{2}}$ is

$$
L E\left(K_{\frac{n-2}{2}}\right)=\left|0-\frac{\frac{2(n-2)(n-4)}{8}}{\frac{n-2}{2}}\right|+\frac{n-4}{2}\left|\left(\frac{n-2}{2}\right)-\left(\frac{\frac{2 n(n-4)}{8}}{\frac{n-2}{2}}\right)\right|=n-4 .
$$

For $K_{2}$, we have $\left|V\left(K_{2}\right)\right|=2,\left|E\left(K_{2}\right)\right|=1$ and the Laplacian eigenvalues of $K_{2}$ are 0 and 2. Hence, $\operatorname{LE}\left(K_{2}\right)=2$. Thus, $L E\left(\Gamma_{D_{2 n}}^{c l}\right)=n-4+2=n-2$.

Theorem 4 Let $Q_{4 n}$ be a generalized quaternion group of order $4 n$, where $n$ is even, $n \geq$ $2, n \in \mathbb{N}$ and let $\Gamma_{Q_{4 n}}^{c l}$ be its conjugacy class graph. Then, the Laplacian energy of $\Gamma_{Q_{4 n}}^{c l}$ is $L E\left(\Gamma_{Q_{4 n}}^{c l}\right)=2 n$.

Proof Consider that $Q_{4 n}$ is the generalized quaternion group of order $4 n$ where $n$ is even, $n \geq 2, n \in \mathbb{N}$ and $\Gamma_{Q_{4 n}}^{c l}$ is its conjugacy class graph. From Proposition 2.2, since $n$ is even, $\Gamma_{Q_{4 n}}^{c l}=K_{n+1}$. Hence,

$$
\begin{aligned}
& \left|V\left(\Gamma_{Q_{4 n}}^{c l}\right)\right|=n+1, \\
& \left|E\left(\Gamma_{Q_{4 n}}^{c l}\right)\right|=\frac{(n+1)(n+1-1)}{2}=\frac{n(n+1)}{2}
\end{aligned}
$$


and the Laplacian eigenvalues of $\Gamma_{Q_{4 n}}^{c l}$ are $\mu=0$ and $\mu=n+1$ with multiplicity $n$. By the definition of the Laplacian energy, we have

$$
L E\left(\Gamma_{G}\right)=\sum_{i=1}^{k}\left|\mu_{i}-\frac{2 m}{n}\right|
$$

where $\mu_{i}$ are Laplacian eigenvalues of $\Gamma_{G}, m$ is the number of edges and $n$ is the number of vertices. Thus, by replacing $\mu$ with $0, n+1$ with multiplicity $n, m$ with $n(n+1)$ and $n$ with $n+1$, the Laplacian energy of $\Gamma_{Q_{4 n}}^{c l}$ is

$$
L E\left(\Gamma_{Q_{4 n}}^{c l}\right)=\left|0-\frac{2 n(n+1)}{\frac{2}{n+1}}\right|+n\left|(n+1)-\frac{2 n(n+1)}{\frac{2}{n+1}}\right|=2 n .
$$

Theorem 5 Let $Q_{4 n}$ be a generalized quaternion group of order $4 n$, where $n$ is odd, $n \geq$ $2, n \in \mathbb{N}$ and let $\Gamma_{Q_{4 n}}^{c l}$ be its conjugacy class graph. Then, the Laplacian energy of $\Gamma_{Q_{4 n}}^{c l}$ is $L E\left(\Gamma_{Q_{4 n}}^{c l}\right)=2 n-2$.

Proof Consider that $Q_{4 n}$ is the generalized quaternion group of order $4 n$ where $n$ is odd, $n \geq 2, n \in \mathbb{N}$ and $\Gamma_{Q_{4 n}}^{c l}$ is its conjugacy class graph. From Proposition 2.2, since $n$ is odd, $\Gamma_{Q_{4 n}}^{c l}=K_{n-1} \cup K_{2}$. Hence,

$$
\begin{aligned}
& \left|V\left(K_{n-1}\right)\right|=n-1, \\
& \left|E\left(K_{n-1}\right)\right|=\frac{(n-1)(n-1-1)}{2}=\frac{(n-1)(n-2)}{2}
\end{aligned}
$$

and the Laplacian eigenvalues of $K_{n-1}$ are $\mu=0$ and $\mu=n-1$ with multiplicity $n-2$. By the definition of the Laplacian energy, we have

$$
L E\left(\Gamma_{G}\right)=\sum_{i=1}^{k}\left|\mu_{i}-\frac{2 m}{n}\right|
$$

where $\mu_{i}$ are Laplacian eigenvalues of $\Gamma_{G}, m$ is the number of edges and $n$ is the number of vertices. Thus, by replacing $\mu$ with $0, n-1$ with multiplicity $n-2, m$ with $\frac{(n-1)(n-2)}{2}$ and $n$ with $n-1$, the Laplacian energy of $K_{n-1}$ is

$$
L E\left(K_{n-1}\right)=\left|0-\frac{2(n-1)(n-2)}{\frac{2}{n-1}}\right|+(n-2)\left|(n-1)-\frac{2(n-1)(n-2)}{\frac{2}{n-1}}\right|=2 n-4 .
$$

For $K_{2},\left|V\left(K_{2}\right)\right|=2,\left|E\left(K_{2}\right)\right|=1$ and the Laplacian eigenvalues of $K_{2}$ are 0 and 2. Hence, $L E\left(K_{2}\right)=2$. Thus $L E\left(\Gamma_{D_{2 n}}^{c l}\right)=2 n-4+2=2 n-2$.

Theorem 6 Let $Q D_{2^{n}}$ be a quasidihedral group of order $2^{n}$ where $n \geq 4, n \in \mathbb{Z}^{+}$and let $\Gamma_{Q D_{2^{n}}}^{c l}$ be its conjugacy class graph. Then, the Laplacian energy of $\Gamma_{Q D_{2^{n}}}^{c l}$ is $L E\left(\Gamma_{Q D_{2^{n}}}^{c l}\right)=2^{n-1}$. 
Proof Consider that $Q D_{2^{n}}$ is the quasidihedral group of order $2^{n}$ where $n \geq 4, n \in \mathbb{Z}^{+}$and $\Gamma_{Q D_{2^{n}}}^{c l}$ is its conjugacy class graph. From Proposition 2.3, $\Gamma_{Q D_{2^{n}}}^{c l}=K_{2^{n-2}+1}$. Hence,

$$
\begin{aligned}
& \left|V\left(\Gamma_{Q D_{2^{n}}}^{c l}\right)\right|=2^{n-2}+1, \\
& \left|E\left(\Gamma_{Q D_{2^{n}}}^{c l}\right)\right|=\frac{\left(2^{n-2}+1\right)\left(2^{n-2}+1-1\right)}{2}=2^{n-3}\left(2^{n-2}+1\right)
\end{aligned}
$$

and the Laplacian eigenvalues of $\Gamma_{Q D_{2^{n}}}^{c l}$ are $\mu=0$ and $\mu=2^{n-2}+1$ with multiplicity $2^{n-2}$. By the definition of the Laplacian energy, we have

$$
L E\left(\Gamma_{G}\right)=\sum_{i=1}^{k}\left|\mu_{i}-\frac{2 m}{n}\right|,
$$

where $\mu_{i}$ are Laplacian eigenvalues of $\Gamma_{G}, m$ is the number of edges and $n$ is the number of vertices. Thus, by replacing $\mu_{i}$ with $0,2^{n-2}+1$ with multiplicity $2^{n-2}, m$ by $2^{n-3}\left(2^{n-2}+1\right)$ and $n$ with $2^{n-3}\left(2^{n-2}+1\right)$, the Laplacian energy of $\Gamma_{Q D_{2^{n}}}^{c l}$ is

$$
\begin{aligned}
L E\left(\Gamma_{Q D_{2^{n}}}^{c l}\right) & =\left|0-\frac{2\left(2^{n-2}+1\right)\left(2^{n-3}\right)}{\left(2^{n-2}+1\right)}\right|+2^{n-2}\left|\left(2^{n-2}+1\right)-\frac{2\left(2^{n-2}+1\right)\left(2^{n-3}\right)}{\left(2^{n-2}+1\right)}\right| \\
& =\left|-2^{n-2}\right|+2^{n-2}\left|\left(2^{n-2}+1\right)-2^{n-2}\right|=2^{n-1} .
\end{aligned}
$$

\section{Conclusion}

In this paper, the general formulas for the Laplacian energy of the conjugacy class graphs of dihedral groups of order $2 n$ are found. For $n \geq 3, n$ and $\frac{n}{2}$ are even integer, $L E\left(\Gamma_{D_{2 n}}^{c l}\right)=n$, while for $n$ even and $\frac{n}{2}$ odd integers, $L E\left(\Gamma_{D_{2 n}}^{c l}\right)=n-2$. We also found the general formulas for the Laplacian energy of the conjugacy class graph of generalized quaternion groups and quasidihedral groups, which are, $L E\left(\Gamma_{Q_{4 n}}^{c l}\right)=2 n-2$ and $L E\left(\Gamma_{Q D_{2^{n}}}^{c l}\right)=2^{n-1}$.

\section{Acknowledgments}

The first author would like to express appreciation toward the partial support of International Doctorate Fellowship (IDF) UTM. The authors would also like to acknowledge Ministry of Education (MOE) Malaysia and Research Management Centre (RMC), Universiti Teknologi Malaysia (UTM) Johor Bahru for the financial funding through the Research University Grant (GUP) Vote No. $13 \mathrm{H} 79$.

\section{References}

[1] Gutman, I. The Energy of Graph. Der. Math. stat. Sekt. Forschungszent Graz. 1978. 103:1-22.

[2] Coulson, C. A., OLeary, B., and Mallion, R. B. Huckel Theory for Organic Chemists. London: Academic Press. 1978.

[3] Gutman, I., and Zhou, B. Laplacian energy of a graph. Linear Algebra and its Applications. 2006. 414(1): 29-37. 
[4] Cvetkovic,D., Rowlinson, P. and Simic, S. K. Signless Laplacian of Finite Graphs. Linear Algebra and its Applications. 2007. 42(1):155-171.

[5] Indulai, G. ,Gutman,I. and Vijayakumar, A. On Distance of Graphs. MATCH Commun. Math. Comput. 2008. 60(1):461-472.

[6] Jooyandeh, M., Kiani, D. and Miirzakhah, M. Incidence Energy of a Graph. MATCH Commun. Math. Comput. 2009. 62(3):561.

[7] Cavers, M., Fallat, S. D. and Kirikland, S. On the Normalized Laplacian Energy and Randic Index. Linear Algebra and its Applications. 2009. 62(3): 561.

[8] Chartrand, G., Linda, L., and Ping, Z. Graphs and Digraphs. CRC Press, Taylor and Francis Group. Boca Raton London New York, 2010.

[9] Teranishi, Y. Subgraphs and the Laplacian Spectrum of a Graph. Linear Algebra and its Applications. 2011. 435: 1029-1033.

[10] Bertram. On Graphs Related to Conjugacy classes of Groups. Bull. London Math.Soc. 1990. 22: 569-575.

[11] Samaila, D., Abba, B. I., and Pur, M. P. On the Conjugacy Classes, Centers and Representation of the Groups Sn and Dn. Int. J. Pure Appl. Sci. Technol. 2013. 15(1): $87-95$.

[12] Rabiha, M., Sarmin, N., and Ahmad, E. The Conjugacy Class Graph of Some Finite Groups and its Energy. MJFAS. 2017. 13(4):659-665.

[13] Beineke, L. W., and Wilson, R. J. Topics in Algebraic Graph Theory, Combinatorics, Probability \& Computing. United States of America: Cambridge University Press. 2007.

[14] Bapat, R. B. Graphs and Matrices. New York: Springer. 2010. 